\section{Plastic commitment}

Lineage commitment is not irreversible. In Science, Busslinger and colleagues show that after commitment to B cell fate, murine pro-B cells can reverse their lineage-restricted pattern of gene expression upon induced deletion of a targeted Pax 5 allele. Pax 5 inactivation in pro-B cells hampers further B cell development both in vitro and in vivo. Yet these Pax5-deficient pro-B cells survive, reverting to a multilineagepotential hematopoietic cell that can reconstitute the T cell compartment of RAG-2 $2^{--}$mice upon adoptive transfer. Thus, Pax 5 expression is required to both initiate and maintain lineage commitment to B cell fate.

Science 297, I I0-II3 (2002)

\section{Fatal recognition by CD3 I}

Phagocytes must distinguish viable cells from apoptotic ones. In Nature, Brown et al. show that leukocytes use the adhesion molecule CD31 (also known as PECAM-1) to sense a cell's viability. Homophilic CD31 interactions between viable human leukocytes flowing under low shear conditions and monolayers of macrophages facilitated both cellular attachment and detachment.

However, apoptotic cells, or those expressing a GPI-linked CD31, attach but cannot be released. CD31 ITIM motifs are critical for detachment, as cells expressing mutant ITIMS (Y663F,Y686F) are also unable to disengage. CD31 expressed by apoptotic cells does not associate with phosphatases SHP-1 and SHP-2, suggesting these interactions transmit the detachment signal. Thus, CD31 signaling discriminates between viable and dying cells.

Nature 4I 8, 200-203 (2002)

\section{Nonredundant Notch I}

Notch signaling is required to direct the common lymphoid progenitor toward the $\mathrm{T}$ cell fate, but inactivation of Notch1 in immature DN3 (stage 3 double-negative) thymocytes has no effect on subsequent $\mathrm{CD} 4^{+}$and $\mathrm{CD} 8^{+}$ T cell development. In Immunity, Radkte and colleagues now show inactivation of Notch1 in immature DN2 thymocytes severely impairs $\alpha \beta$, but not $\gamma \delta$, T cell development. Notch1-deficient thymocytes are blockaded at the early DN3 stage, due to impaired $V_{\beta}-D_{\beta} J_{\beta}$ rearrangement, and cause an accumulation of intracellular TCR $\beta$ - DN4 cells. Thus, Notch1 signaling has important nonredundant functions during early $\alpha \beta$ T cell development.

Immunity 16, 869-879 (2002)

\section{Twisted development}

Genes involved in embryonic development continue to reveal functions beyond the orderly formation of body parts. In the Journal of Experimental Medicine, Graf et al. report that the proteins twisted gastrulation (Tsg), bone morphogenetic proteins (BMPs) and chordin are not only involved in embryogenesis, but may be important in thymocyte development. Thymic stromal cells express BMP2(4) and their extracellular inhibitor chordin. BMP2(4) can block thymocyte maturation by interfering with pre-TCR and TCR signaling, thus preventing $\mathrm{CD}^{-} \mathrm{CD}^{-} \rightarrow \mathrm{CD}^{+}{ }^{+} \mathrm{CD}^{+}$and $\mathrm{CD} 4^{+} \mathrm{CD} 8^{+} \rightarrow$ single positive checkpoint progression. The inherent instability of chordin tips the balance such that BMP effects dominate. However, Tsg, which is expressed by thymocytes, synergizes with chordin to overcome the inhibitory signals to thymocyte development from BMP. The balance of expression between these proteins may be one of the myriad control mechanisms in thymopoiesis.

J. Exp. Med. 8 July 2002 (doi: 10.1084/jem.20020276)

\section{IL-I 3-responsive epithelia}

IL-13 production by $\mathrm{T}_{\mathrm{H}} 2$ cells contributes to asthma pathogenesis. Blocking IL-13 inhibits allergen-induced airway hyperreactivity (AHR), whereas delivery of IL-13 to the airway causes AHR, mucus production and eosinophilia in animal models of allergic asthma. However, the identity of the IL13-responsive cells is unknown. In Nature Medicine, Kuperman et al. use STAT6-deficient mice to reveal the identity of the IL13-responsive cells in the lung. STAT6-deficient mice were protected from all pulmonary effects of IL-13. However, reconstitution of only epithelial cells with STAT6 no longer protected mice from IL-13-induced AHR. Thus, IL-13 directly effects airway epithelial cells to cause AHR and mucous overproduction. Blocking the IL-13 receptor or downstream signaling pathways could therefore potentially improve the specificity of asthma treatment.

Nature Medicine 8, 885-889 (2002)

\section{Exposing CD3\& to Nck}

How antigen engagement triggers $\mathrm{T}$ cell activation is one of the most intensely studied immune events. In Cell, Gil et al. show ligand binding by TCRs induces a conformational change in $\mathrm{CD} 3 \varepsilon$, which exposes a proline-rich cytoplasmic domain to the adaptor protein Nck. Contrary to previous TCR signaling models that place Nck downstream from SLP-76 in the signaling cascade, Nck binds to the TCR-CD3-ligand complex before tyrosine phosphorylation of receptor ITAM motifs by protein tyrosine kinases. Blocking this Nck-CD3 $\varepsilon$ interaction inhibits subsequent $\mathrm{T}$ cell activation, presumably by disrupting signals required for cytoskeletal reorganization to bring about immune synapse formation. Thus, ligand binding triggers two independent TCR signaling events that are required to activate $\mathrm{T}$ cells: direct recruitment of Nck to the TCR by $\mathrm{CD} 3 \varepsilon$ and phosphorylation by protein tyrosine kinases.

\section{Cell 109, 90I-912 (2002)}

\section{Death-by-PARP-AIF}

Poly (ADP-ribose) polymerase-1 (PARP-1) is an abundantly expressed enzyme in the nucleus that mediates DNA repair during homeostasis and contributes to cell death after stress and injury. Activation of PARP-1 leads to the depletion of $\mathrm{NAD}^{+}$and ATP, conditions that favor necrosis, but the molecular mechanisms of PARP-1-induced cell death are unknown. Yu et al. report in Science that stress-activated PARP-1 induces a characteristic apoptotic cell death, with release of cytochrome $\mathrm{c}$, mitochondrial membrane depolarization and annexin $\mathrm{V}$ staining. PARP-1 activation triggers the translocation of AIF into the nucleus, leading to chromatin condensation and the initiation of apoptosis. Although PARP-1 activation also induces caspase expression, inhibitors of caspases do not block the apoptotic process. By mediating both necrosis and apoptosis, PARP-1 may be an important component of most cell death.

Science 297, 259-263 (2002) 